\title{
Plenary Presentations
}




\title{
Pulsars are cool. Seriously.
}

\author{
Scott M. Ransom \\ NRAO, 520 Edgemont Road, Charlottesville, VA 22903, USA \\ email: sransom@nrao.edu
}

\begin{abstract}
Ever since the first pulsar was discovered by Bell and Hewish over 40 years ago, we've known that not only are pulsars fascinating and truly exotic objects, but that we can use them as powerful tools for basic physics and astrophysics as well. Taylor and Hulse hammered these views home with their discovery and timing of the spectacular "binary pulsar" in the 1970s and 1980s. In the last two decades a host of surprises and a promise of phenomenal scientific riches in the future has come from the millisecond pulsars. As our instrumentation has become more sensitive and better suited to measuring the pulses from these objects, they've given us new tests of general relativity, fantastic probes of the interstellar medium, constraints on the physics of ultra-dense matter, new windows into binary and stellar evolution, and the promise of a direct detection of gravitational waves. These things really are cool, and there is much more we will do with them in the future.
\end{abstract}

Keywords. pulsars: general, words: superlatives and colloquialisms

Pulsars really are cool. Not in the temperature sense, given that their surfaces are at about a million kelvin, but in the other. These are city-sized (10-12 km radii) neutron stars with up to twice the mass of our Sun. Their central densities are several times higher than atomic nuclei, so high that our current nuclear and particle physics cannot accurately predict what goes on deep within these stars. They have surface gravities 100 billion times stronger than the Earth's, making nearby space-time highly curved. Their magnetic fields range from 100 million times to a quadrillion times stronger than the Earth's — fields so strong that quantum effects become important (see Nanda Rea's contribution to these proceedings). They can spin over 700 times per second, which is faster than racing car engines rotate and kitchen blenders spin. They emit electromagnetic radiation via detailed processes we don't understand after over 40 years of hard work and at luminosities, coming only at the expense of rotation(!), of up to 10,000 times more than the total output of the Sun. There is no denying that these are exotic objects.

Yet even if we ignore their exoticness, they are still cool. The stories of the original pulsar discovery by Jocelyn Bell and Tony Hewish (Hewish et al. 1968) and of the first binary pulsar by Joe Taylor and Russell Hulse (Hulse \& Taylor 1975), and the ensuing Nobel glories and gaffes, are the stuff of astronomical legend. For me though, what really makes pulsars so cool is how they can be used as tools for a wide variety of physics and astrophysics problems by the miracle of pulsar timing. The "stars" of these measurements are most certainly the millisecond pulsars (MSPs).

\section{Millisecond pulsars}

Millisecond pulsars are distinct from the $~ 2000$ "normal" pulsars known in that they have been "recycled" (Alpar et al. 1982 and Radhakrishnan \& Srinivasan 1982). A pulsar is born in a supernova and then radiates and spins-down as a normal (i.e. $\sim 1$ second spin period) pulsar for 10-100 Myr. If the system was in a binary which survives the supernova though, the secondary star will evolve on Gyr timescales and, when it begins to ascend the 
giant branch, transfers mass and angular momentum onto the long-dead pulsar. During this period the system is observable as an X-ray binary. When the stellar and binary evolution finishes, an MSP emerges, spinning hundreds of times per second in a nearly perfectly circular orbit around a white dwarf. Since the neutron star's magnetic field is somehow buried by the accretion, from $10^{12}$ Gauss down to $10^{8}$ Gauss, the new MSP spins down much more slowly, providing us with a nearly perfect clock visible for billions of years.

\section{Pulsar timing}

At 8:40AM CST, on August 23, 2012 (which was when I was at this point in my talk at the IAU in Beijing), the spin period of one of the best timed MSPs, J0437-4715, was exactly $5.7574518556687 \mathrm{~ms}$ with an error of \pm 1 in the last digit. Since the pulsar loses rotational energy due to its emission of a relativistic wind and electromagnetic radiation, that last digit increases odometer-like by one every half hour. That means that the first 6 digits will remain constant for about the next millennium! This stability, and our ability to measure it via pulsar timing, is why pulsars enable truly revolutionary measurements.

Pulsar timing is really quite simple in concept. We unambiguously account for each and every rotation of a pulsar over a time span of years. In practice, we can actually track small fractions of each rotation, thereby making pulsar timing a precise form of phase measurement. With the start of an observation referenced via GPS to worldwide atomic time standards and time during each observation tracked using hydrogen masers at the observatories, we can measure the average times of arrival (aka TOAs) of MSP pulses to better than $1 \mu \mathrm{s}$, which corresponds to about $10^{-4}$ in rotational phase $\phi$. Since an error in frequency is simply $\Delta \phi / \Delta T$, if we can make measurements like this over time spans $\Delta T$ of 3 years (i.e. $10^{8}$ seconds), our measurement error in the spin frequency of a pulsar is $10^{-12} \mathrm{~Hz}$, corresponding to about 14 significant figures for MSPs.

We establish timing "solutions" for pulsars via a bootstrapping series of observations scheduled such that we never lose count of the number of rotations our target pulsar makes. A dense set of observations involving several over one or two days, and then several more spaced over the next week allow us to solve binary orbital parameters and determine an increasingly precise spin frequency. As more time is added to the timing solution, the Earth's orbital motion allows us to determine highly-precise (down to tens of micro-arc-seconds for MSPs) astrometric positions and eventually even proper motions of the pulsars, and the spin-down of the pulsar appears as an increasing quadratic delay in the pulse arrival times. After a year, the timing solution is complete, and it includes a precise position, spin-frequency, and spin-down rate, and many significant figures in the five Keplerian orbital parameters if the pulsar is in a binary (orbital period, projected semi-major axis, eccentricity, time of periastron passage, and the argument of periastron). Often characterized by the root-mean-squared (RMS) deviation of the timing residuals (i.e. TOAs minus model predictions), state-of-the-art timing solutions are below 100 nano-seconds RMS over timescales of 5 or more years.

Published MSP timing solutions are some of the most precise measurements in all of astrophysics and have enabled science which would be effectively impossible using other techniques. A great example is the fact that MSP timing provides pulsar radial velocity measurements at ridiculous precisions of a few $\mathrm{mm} / \mathrm{s}$ compared to $\sim 1 \mathrm{~m} / \mathrm{s}$ for optical radial velocity planet surveys. That precision allowed Wolszczan \& Frail (1992) to uncover the first extrasolar planets around MSP B1257+12. Those planets are all among the lowest mass exoplanets yet detected, and planet "A" is only twice the mass of the Moon! 


\section{A millisecond pulsar renaissance}

Over the past decade, and especially over the past 5 years, the pulsar field has been focused on MSPs. The simple reason for this is Moore's Law, since computation and the improved digital instrumentation based on it has dramatically improved our ability to discover and time MSPs. We need to sample our radio data at rates much faster than the already rapid spin rates of MSPs in order to make precise measurements of them. Effective sampling times of $\sim 50 \mu$ s are now the standard for pulsar searches and times below $1 \mu$ s are common for high-precision timing observations.

In addition, since radio pulses propagate through the ionized interstellar medium, they experience dispersion such that the lower radio frequencies $\nu$ are delayed quadratically (i.e. $\Delta t \propto \nu^{-2}$ ) with respect to the high frequencies. To compensate, we divide our observing band into many independent frequency channels, effectively making spectra for each sample in time, and then delay the channels appropriately so that the pulses sum in phase. $\dagger$ For modern pulsar surveys, which are (finally) nearly as sensitive to MSPs as they are to normal pulsars, we require thousands of spectral channels across our observing bands. Combined with fast sampling, data rates of $\sim 50 \mathrm{MB} / \mathrm{s}$ are common for each pixel in the surveys, and total data volumes can comprise nearly a Petabyte. Computing is extremely important and costly for search processing as well since we must search over thousands of independent "Dispersion Measure" (aka DM) trials since the amount of dispersion is unknown a priori for a new pulsar $\ddagger$.

Finally, since pulsars are intrinsically faint but continuum radio sources, we want large observing bandwidths to integrate over in order to maximize the signal-to-noise of our detections. In general, they have steep radio spectra which limits the highest useful observing frequencies to $\sim 3 \mathrm{GHz}$, while radio interference, interstellar scattering, and the Galactic synchrotron background limit low frequencies to $\sim 300 \mathrm{MHz}$. Custom state-ofthe-art digital instrumentation is required to rapidly sample and channelize bandwidths of hundreds to thousands of $\mathrm{MHz}$ and cluster computing is required to process it.

The advances of Moore's Law over the last decade have resulted in pulsar instrumentation which has been asymptotically approaching perfection given the $\sim 3 \mathrm{GHz}$ of total bandwidth available for pulsar observations. For the first time ever, we are being limited by the sizes of our telescopes rather than the capabilities of the radio receivers or our pulsar instrumentation. The new instrumentation has brought new life to "classic" single-dish radio telescopes used for pulsar observations such as Parkes, Jodrell Bank, and Arecibo, and will finally allow us to use "new" telescopes such as the GBT to their fullest.

Besides dramatically improved sensitivities to new MSPs in the current generation of pulsar surveys, our ability to time MSPs has increased in a Moore's Law fashion as well. In the 30 years since the discovery of the first MSP by Backer et al. (1982), the typical timing precision for high-precision pulsars has improved by a factor of several hundred, from RMSs of 10 s of microseconds to better than $\sim 100$ ns. Such timing precision has opened up completely new probes of physics.

$\dagger$ For pulsar timing, we can Nyquist-sample the full observing band and perform what is known as coherent de-dispersion to exactly remove the dispersive delays. This technique is incredibly computationally intensive and has only recently become feasible across large observing bandwidths.

$\ddagger$ Most modern surveys also perform tens to hundreds of so-called "acceleration" trials for each DM trial to improve sensitivities to interesting pulsars in compact binary systems. 


\section{Case in point: MSP J1614-2230}

A beautiful example of how instrumentation can so dramatically change what is possible in pulsar observing is the MSP J1614-2230. It was uncovered in a last-generation survey of EGRET gamma-ray error boxes (Crawford et al. 2006) and appeared to be a fairly "vanilla" MSP with a spin period of $3.15 \mathrm{~ms}$ in an 8.7-day circular orbit with a white dwarf. Because of its position in the sky, it was a perfect "test" pulsar to observe for a minute or two to check the GBT's observing system before starting regularly scheduled long-term observations of MSPs in globular clusters near the Galactic center. After accumulating almost 5 years of not-very-good (i.e. $\sim 10 \mu$ s RMS) timing data in this manner, we noticed systematic delays during a small portion of the orbit when the pulsar passed behind the companion star (i.e. superior conjunction). On three separate days, the pulses arrived later than expected by $20-40 \mu \mathrm{s}$. We knew from the orbital parameters and the Keplerian mass function that the companion star was at least $0.4 \mathrm{M}_{\odot}$, which is quite massive compared to most other MSP companions (which are typically $\left.0.1-0.2 \mathrm{M}_{\odot}\right)$.

We conjectured that the systematics were due to the "Shapiro Delay" of the pulses as they passed through the gravitational potential of the white dwarf. Irwin Shapiro first identified this effect in 1964 and then measured it with beautiful radar experiments in the late 1960's and early 1970's (e.g. Shapiro et al. 1971). We had just built a brand-new wideband pulsar instrument for the GBT called GUPPI $\dagger$ (Green Bank Ultimate Pulsar Processing Instrument), based on field programmable gate arrays (FPGAs) and high-end graphics processing units (GPUs) made for computer gaming. GUPPI can effectively perfectly process the full $800 \mathrm{MHz}$ bandwidth of the GBT's L-band receiver, a factor of more than 10 increase in what was possible with the previous generation high-precision pulsar backend at the GBT. If we used it in a week-long observing campaign, with observations of several hours each day, we predicted that the timing precision would increase by a factor of up to 10 .

The 8-hour observation during conjunction was simply stunning. Hundreds of data points with $\sim 1 \mu$ s errors showed the extremely strong and cusp-like signature indicative of Shapiro delay from a nearly edge-on orbit. When the observing campaign was over, we measured the two parameters associated with the Shapiro delay to high precision, which in combination with the Keplerian orbital parameters, gave us the mass of the white dwarf $\left(0.500 \pm 0.006 \mathrm{M}_{\odot}\right)$, the orbital inclination $(89.17 \pm 0.02$ degrees!), and a pulsar mass of $1.97 \pm 0.04 \mathrm{M}_{\odot}$, by far the most massive precisely measured neutron star to date (Demorest et al. 2010). The GBT with GUPPI had turned a "vanilla" MSP into an important probe of high-density physics, which has strongly constrained the neutron star equation of state (i.e. EOS; Lattimer \& Prakash 2010) and touched on many other aspects of both basic and astro-physics (e.g. Özel et al. 2010). It also made J1614-2230 into a timing array pulsar for the detection of gravitational waves.

\section{Gravitational waves: the next frontier for MSPs?}

Pulsar timing arrays (PTAs) have the potential to help revolutionize our view of the Universe, by giving us direct detections of gravitational waves (GWs), and maybe (just maybe) doing it before Advanced LIGO does. The idea of using pulsar timing to detect gravitational waves goes back to Detweiler (1979). The basic gist is that GWs with wavelengths of light-years, or consequently frequencies in the nanohertz regime, will stretch and compress the space-time through which radio pulses travel and thereby advance or

$\dagger$ https://safe.nrao.edu/wiki/bin/view/CICADA/NGNPP 
delay their arrival times here at Earth. A problem, though, is that long-term changes in arrival times from a pulsar could be due to a variety of reasons, such as errors in our atomic time-standards or planetary ephemerides, or even simply timing noise from the pulsar itself. Hellings \& Downs (1983) though, showed that the quadrupolar-nature of GWs leads to correlated delays in the arrival times from an array of pulsars, based on the angular separation of pairs of pulsars on the sky.

Where would these GWs come from? While it is possible that we could see "strong" GWs left over from Inflation or from the interaction of cosmic strings, most people believe our most likely sources of nanohertz GWs will come from supermassive $\left(10^{8}-10^{9} \mathrm{M}_{\odot}\right)$ black hole binaries (SMBHBs) orbiting on years-long timescales before they coalesce. Such binary systems are thought to exist throughout the Universe as a result of galaxy mergers during hierarchical structure formation (e.g. Sesana 2012). These black hole binaries, even at distances of a Gpc, can cause perturbations of order $10 \mathrm{~ns}$ in pulsar timing residuals. If Nature was kind to us, the strongest of these sources (meaning a combination of the most massive and closest to us in the nanohertz frequency regime), will be detected individually. Such a detection could allow for localization on the sky, detection in other electro-magnetic wavebands, and might allow us to "calibrate" and improve our PTAs similar to the way that interferometers calibrate and "phase-up" on bright astrophysical point sources.

Even without a strong nearby individual source, though, we are likely to detect a steepspectrum (i.e. much stronger at lower GW frequencies) stochastic background made up of the ensemble of all SMBHBs throughout the Universe (e.g. Hellings \& Downs 1983), within the next 5-10 years. One of the great things about the stochastic background is that as we time the pulsars in our PTAs longer, and therefore to lower frequencies, our sensitivity increases not just by simply accumulating more data, but by climbing up the steep spectrum to where the GWs are stronger. This results in our sensitivities improving at a much faster rate than the naive $\sqrt{T}$. No matter what, the signal amplitudes will only be in the nanoseconds to tens of nanoseconds regime and so we need the best instrumentation and the best MSPs for our experiments.

Currently there are three major PTA efforts underway: NANOGrav, the North American Nanohertz Observatory for Gravitational Waves, which uses Arecibo and the GBT (Demorest et al. 2012); the European Pulsar Timing Array (EPTA), using several of Europe's largest telescopes (van Haasteren et al. 2011); and the Parkes Pulsar Timing Array (PPTA), using the Parkes telescope in Australia (Manchester et al. 2012). Each of these efforts has been timing $\sim 15-30$ MSPs for the past 5-10 years with continually improving timing residuals, including several pulsars in the 50-100 ns regime and many more at $100-300 \mathrm{~ns}$. In addition, there is an effort underway to combine the data from all three PTAs into the International Pulsar Timing Array (IPTA; Hobbs et al. 2010), which will improve the overall sensitivity by a factor of $\sim 2$ compared to any single PTA.

Despite the fact that PTA sensitivities are continuing to get better due to improvements in our timing techniques and our instrumentation, we still need more and better MSPs to fully achieve the potential of a pulsar-based GW "observatory". That potential, and the possibility of an imminent GW detection by pulsar timing, is driving many large-scale efforts to find more MSPs.

\section{New surveys for millisecond pulsars}

The millisecond pulsar renaissance can most certainly be seen in the results of the recent and ongoing surveys for new pulsars. New instrumentation and increased compute capacity has dramatically improved MSP search sensitivities from the same telescopes 

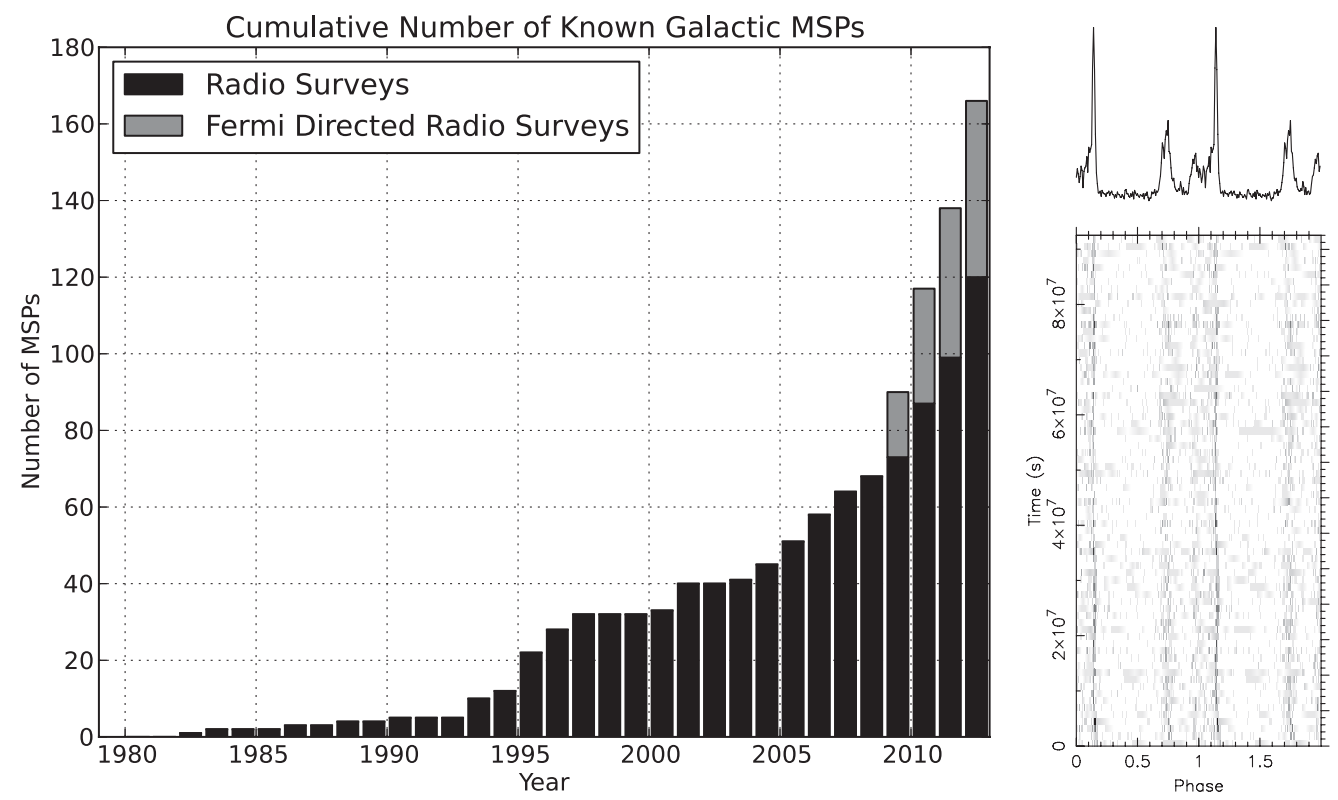

Figure 1. (Left) Number of known Galactic (i.e. not in globular clusters) millisecond pulsars (MSPs) as a function of year, through November 1, 2012. MSPs are defined here as recycled pulsars spinning faster than $15 \mathrm{~ms}$. The rapidly increasing numbers of these systems are due primarily to new wide-area radio surveys using much improved instrumentation and Fermi's ability to point us at likely radio MSPs. For up-to-date numbers, see Duncan Lorimer's list at http://astro.phys.wvu.edu/GalacticMSPs/GalacticMSPs.txt (Right) Folded Fermi LAT gamma-ray photons of the $3.68 \mathrm{~ms}$ radio and gamma-ray binary MSP J1231-1411 (Ransom et al. 2011). The plot contains 3 yrs of Fermi data, corresponding to $\sim 3000$ photons (about 3 per day!), $\sim 560$ binary orbits, and 24 billion rotations of the pulsar, yet the main peak has a width of just over $2 \%$ of the pulse period - a beautiful example of the power of pulsar timing.

and even from the same radio receivers which have surveyed the sky before. The number of Galactic MSPs (i.e. those not in globular clusters, whose numbers have increased hugely as well) has doubled in the last three years and quadrupled in the last decade (see Figure 1). About half of those found in the past few years have come from a series of largearea surveys being conducted by the GBT (the GBT Driftscan and Green Bank North Celestial Cap or GBNCC surveys; Lynch et al., these procs.), Arecibo (Pulsar-ALFA and the AO327 Driftscan surveys; Lazarus et al., these procs.), and Parkes (the three related High Time Resolution Universe or HTRU surveys; Keith et al., these procs.).

These surveys are using either low-frequency $(\sim 350 \mathrm{MHz})$ receivers with short dwell times or higher-frequency systems $(\sim 1400 \mathrm{MHz})$ with multiple beams to increase survey speeds and enable them to cover large areas of the sky. When complete in the next couple years, the full sky will have been re-surveyed by one or more telescopes and the total data volume will approach 2 Petabytes. While the data-taking is not quite halfway complete, an even smaller fraction of the new data has been fully processed, ensuring that the discovery rate will continue for several years to come.

A fantastic short-cut to finding new MSPs has been provided by the Fermi satellite, though. Shortly after launch, Fermi showed that most MSPs are copious producers of pulsed gamma-ray emission (Abdo et al. 2009). That meant that many of the unassociated Fermi LAT sources, especially those well off of the Galactic plane, might be MSPs. A collaboration of radio astronomers working with the LAT team, called the Pulsar Search 
Collaboration, has uncovered at least 45 new radio MSPs (and counting!) by searching these gamma-ray sources deeply with the biggest radio telescopes around the world (Ray et al. 2012). We would eventually find most of these MSPs with increasingly sensitive all-sky radio surveys, but Fermi is showing us exactly where to look and allowing us to find them much sooner. Once radio timing solutions are established, basically all of them are seen to pulse in gamma-rays as well (see Figure 1), giving us a brand new probe into the pulsar magnetosphere and emission processes. A large fraction of the new Fermi MSPs are turning out to be previously rare eclipsing systems, a point we currently do not understand. Since there seems to be no strong correlation between the gamma-ray and radio fluxes, we expect that new Fermi-directed radio MSPs will be uncovered for as long as new Fermi sources are being detected.

Most pulsar surveys uncover one or two surprises, and in fact it is for these exotic systems that we often tune the parameters of our searches. High on the current "Most Wanted" list are a sub-millisecond pulsar, which would strongly constrain the EOS of neutron star matter, and a pulsar-black hole system, which would be an incredible testbed of strong-field general relativity (see Michael Kramer's contribution to these proceedings). We don't have those yet(?), but there have certainly been other recent surprises.

The GBT Driftscan survey uncovered a fast and bright MSP in a 4.75-hr orbit which, it turned out, had been studied as a likely accreting cataclysmic variable, in the optical, radio, and X-rays for the decade preceding its discovery as an MSP (Archibald et al. 2009). The pulsar, J1023+0038, seems to be a "Missing Link" system in the late stages of the recycling process as stellar evolution of the evolved companion nears completion. There are now a half dozen similar systems known, many uncovered with the help of Fermi, and all fascinating probes into binary and stellar evolution $\dagger$ (Roberts, these procs.).

Deep in the Galactic plane, the P-ALFA survey uncovered another evolutionary oddball, MSP J1903+0327, which is in an eccentric orbit around a Sun-like main-sequence star (Champion et al. 2008). This highly-inclined system can be timed quite precisely and has yielded Shapiro delay as well as the relativistic orbital precession of periastron, thereby providing a very precise, and fairly high, neutron star mass $\left(1.667 \pm 0.021 \mathrm{M}_{\odot}\right.$; Freire et al. 2011). The formation mechanism of the system is uncertain, but given that the star which spun up the pulsar is missing, a likely triple scenario involving a dynamical instability seems to be the best bet. Intriguingly, the GBT Driftscan survey has recently uncovered an MSP which is currently in a triple stellar system (Ransom et al. in prep.).

Finally, more pulsar planets, or at least planet-mass companions, have been uncovered in three new MSP systems, two of which were announced at this conference (see Bates et al. and Lynch et al.). The first of these is the so-called "Diamond Planet" system J1719-1438, with a Jupiter-mass companion in a compact 2.2-hr binary (Bailes et al. 2011). The density of the companion is constrained to be very large, implying that it is an ultra-low mass carbon white dwarf in crystalline form (i.e. diamond!). Pulsar timing can easily detect planets of almost any reasonable mass, so it is interesting to ask why are there so few pulsar planets?

\section{Prospects for the future}

With pulsar instrumentation approaching perfection and several large surveys underway and already successful, it is easy to predict good things from pulsars, and in particular MSPs, in the coming few years. We will eke out additional timing precision from our instruments and techniques which will lead to more surprises, and potentially on

$\dagger$ These systems have been coined "Redbacks" in spider-salute to the "Black Widow" pulsars. 
short timescales - more planets? more massive or maybe low-mass neutron stars? gravitational waves? And all-sky or targeted surveys could uncover a new "Holy Grail" any day, an eccentric MSP-MSP binary could lurk in a globular cluster, and SgrA* should be surrounded by hundreds of pulsars.

Unfortunately, though, there are some issues. Nearly all of the "classic" pulsar telescopes, Arecibo, Jodrell Bank's Lovell Telescope, Parkes, and now the GBT, have recently been or are currently under serious threat of closure due to dwindling or changing budgets. "Simple" single-dish radio telescopes can do fantastic things for pulsar astronomy, but they are being eclipsed by current and next-generation radio arrays which promise to do more things for more astronomers. Great pulsar astronomy will certainly be done with upcoming arrays like MeerKAT, LOFAR, and the Phase I SKA, but things are trickier and potentially costlier with arrays. It will be important to carefully weigh the costs of closing simple single-dish telescopes, which can be very effective at producing high-impact pulsar science, as we march towards the era of giant radio arrays.

China's upcoming 500-m diameter single-dish called FAST will be an excellent test case (Li et al., these procs.). Its incredible sensitivity and increased sky coverage compared to Arecibo could revolutionize pulsar astronomy before the Phase I SKA is even partially complete. No matter what, if and when these giant new facilities come on line, some of the first and best science you will see will be from pulsars. How could it not? These things are wicked cool. Seriously.

\section{References}

Abdo, A. A., Ackermann, M., Ajello, M., et al. 2009, Science, 325, 848

Alpar, M. A., Cheng, A. F., Ruderman, M. A., \& Shaham, J. 1982, Nature, 300, 728

Archibald, A. M., Stairs, I. H., Ransom, S. M., et al. 2009, Science, 324, 1411

Backer, D. C., Kulkarni, S. R., Heiles, C., Davis, M. M., \& Goss, W. M. 1982, Nature, 300, 615

Bailes, M., Bates, S. D., Bhalerao, V., et al. 2011, Science, 333, 1717

Champion, D. J., Ransom, S. M., Lazarus, P., et al. 2008, Science, 320, 1309

Crawford, F., Roberts, M. S. E., Hessels, J. W. T., et al. 2006, ApJ, 652, 1499

Demorest, P. B., Pennucci, T., Ransom, S. M., Roberts, M. S. E., \& Hessels, J. W. T. 2010, Nature, 467, 1081

Demorest, P. B., Ferdman, R. D., Gonzalez, M. E., et al. 2012, ApJ, in press (arXiv:1201.6641)

Detweiler, S. 1979, ApJ, 234, 1100

Freire, P. C. C., Bassa, C. G., Wex, N., et al. 2011, MNRAS, 412, 2763

Hellings, R. W. \& Downs, G. S. 1983, ApJ, 265, L39

Hewish, A., Bell, S. J., Pilkington, J. D. H., Scott, P. F., \& Collins, R. A. 1968, Nature, 217, 709

Hobbs, G., Archibald, A., Arzoumanian, Z., et al. 2010, Class. E Quant. Grav., 27, 084013

Hulse, R. A. \& Taylor, J. H. 1975, ApJ, 195, L51

Lattimer, J. M. \& Prakash, M. 2010, (arXiv:1012.3208)

Manchester, R. N., Hobbs, G., Bailes, M., et al. 2012, PASP in press, (arXiv:1210.6130)

Özel, F., Psaltis, D., Ransom, S., Demorest, P., \& Alford, M. 2010, ApJ, 724, L199

Radhakrishnan, V., \& Srinivasan, G. 1982, Current Science, 51, 1096

Ransom, S. M., Ray, P. S., Camilo, F., et al. 2011, ApJ, 727, L16

Ray, P. S., Abdo, A. A., Parent, D., et al. 2012, (arXiv:1205.3089)

Sesana, A. 2012, Adv. in Astro., 2012, \#805402

Shapiro, I. I. 1964, Phys. Rev. Lett., 13, 789

Shapiro, I. I., Ash, M. E., Ingalls, R. P., et al. 1971, Phys. Rev. Lett., 26, 1132

van Haasteren, R., Levin, Y., Janssen, G. H., et al. 2011, MNRAS, 414, 3117

Wolszczan, A. \& Frail, D. A. 1992, Nature, 355, 145 